\title{
Potential and limitations of nuclear magnetic resonance for the cardiologist
}

\author{
GEORGE K RADDA \\ From the University Department of Biochemistry and the Clinical Magnetic Resonance Laboratory, Radcliffe \\ Infumary, Oxford
}

One of the fundamental problems in cardiovascular research is concerned with the relation between biochemical energy provision and demand of the myocardium in healthy and diseased states. Until recently most of the available biochemical information was a result of indirect or highly invasive measurements. During the past nine years we have shown that nuclear magnetic resonance spectroscopy is a powerful tool for the study of tissue energetics ${ }^{1}$ and have developed techniques for the study of isolated and in vivo organs in animals ${ }^{2}$ and more recently in man. ${ }^{3}$

Nuclear magnetic resonance is observed as a very weak response when chemical substances are placed in a strong uniform magnetic field and are irradiated with weak radiofrequency waves. It arises from the interaction of atomic nuclei with the applied magnetic field and yields detailed information about chemical structures and molecular motions. Only those nuclei that have magnetic properties give rise to signals. For example, when the ${ }^{1} \mathrm{H}$ atom is observed signals mainly from water and fat are obtained and are used for reconstructing spatially resolved images. The clinical applications of nuclear magnetic resonance imaging are reviewed in this issue. ${ }^{4}$ In our own work we use nuclear magnetic resonance in a different way. If we apply considerably stronger and more homogeneous magnetic fields than those used in imaging signals from individual chemicals can be resolved in the frequency domain. We have mainly concentrated on detecting phosphorus nuclei and are now able to follow various biochemical processes by the use of phosphorus ( $\left.{ }^{31} \mathrm{P}\right)$ nuclear magnetic resonance spectroscopy. To appreciate the clinical value of the method in cardiology we should consider the bioenergetic processes in muscle (Fig. 1).

The key role of adenosine triphosphate in providing the energy for work is assured by adequate provisions for maintaining its concentration through three sepa-

Based on the British Heart Foundation prize lecture delivered at the meeting of the British Cardiac Society, 7 December 1982

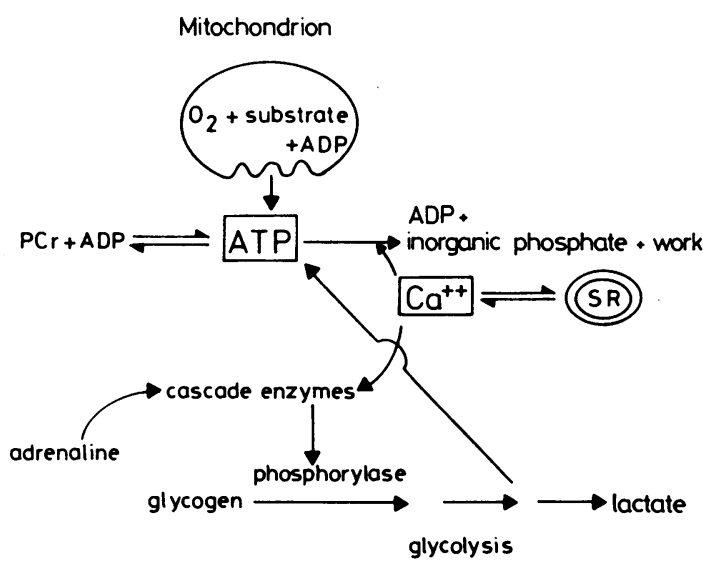

Fig. 1 Energetics in heart and muscle ( $A D P$, adenosine diphosphate; $P C r$, phosphocreatine; $A T P$, adenosine triphosphate).

rate but closely interlinked reactions: (a) oxidative phosphorylation, (b) the rapid conversion of phosphocreatine to adenosine triphosphate, and (c) anaerobic degradation of glycogen to lactic acid. The fluxes through the different pathways are controlled in several ways and the switch from aerobic to anaerobic production of adenosine triphosphate involves a complex series of reactions catalysed by a cascade of enzymes in which $\mathrm{Ca}^{++}$has a special role. Lactate production is accompanied by cellular acidification, particularly under conditions of restricted flow, and as a result is believed to contribute to irreversible damage during cardiac ischaemia.

In my view it is inconceivable that knowledge of the concentrations of high energy phosphates, the fluxes through the various reactions shown in Fig. 1, and the way these are controlled would not be useful to the clinician. ${ }^{31} \mathrm{P}$ nuclear magnetic resonance is now at a stage where such information can be obtained noninvasively from humans. I shall summarise some of our work under three main headings. 


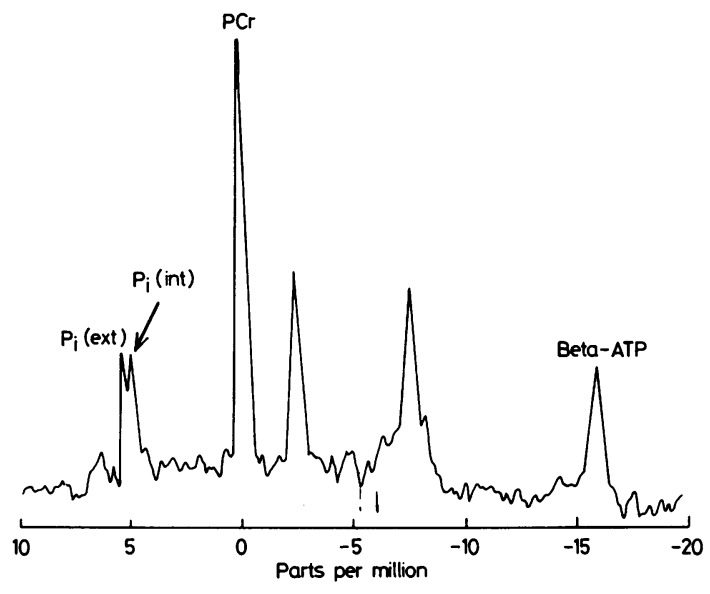

Fig. $2{ }^{31} P$ nuclear magnetic resonance spectrum of perfused rat heart. Peaks from left to right: $P_{i(e x t)}$, extermal inorganic phosphate (indicating $p H$ of 74); $P_{i(i m)}$, intermal inorganic phosphate (indicating pH of 7.03); PCr: phosphocreatine; beta ATP, beta phosphate group of adenosine triphosphate.

Nature of information from ${ }^{31} \mathrm{P}$ nuclear magnetic resonance

\section{CONCENTRATIONS OF METABOLITES AND INTRACELLULAR $\mathrm{pH}$}

Fig. 2 shows a typical ${ }^{31} \mathrm{P}$ nuclear magnetic resonance spectrum obtained from an isolated perfused rat heart. The chemical responsible for each resonance can be identified from the frequency of the signal while the area under each peak gives a direct measure of the amount of the corresponding compound present in the sample. We can also find out something about intermolecular interactions, environment, and molecular motion from measurements of line widths and relaxation indices. Simultaneously, from the position of the signal from inorganic phosphate we can derive the value of intracellular cytoplasmic $\mathrm{pH}$.

For example, during cardiac arrest (which we can simulate in the isolated heart by inducing total global ischaemia) we observe the rapid depletion of phosphocreatine (in less than 4 minutes at $37^{\circ} \mathrm{C}$ ), the build up of inorganic phosphate, and intracellular acidification. Decrease in adenosine triphosphate is observed only after all the phosphocreatine has been used up. The time course of changes in metabolites can thus be rapidly followed both in the early stages of ischaemia and during recovery brought about by reperfusion. ${ }^{5}$ During a 12 to 15 minute period of total global ischaemia intracellular $\mathrm{pH}$ decreases from 7.05 to 6.4. It has coten been suggested that high acidity can lead to irreversible damage during ischaemia. Our studies suggest that while the ischaemic myocardium can be protected by good external buffering, which reduces intracellular acidosis, ${ }^{6}$ low $\mathrm{pH}$ alone does not account for irreversible damage. For example, in hearts treated with insulin before global ischaemia, intracellular pH decreases to as low a value as 5.9 yet functional and metabolic recovery is observed after reflow. This is because adenosine triphosphate is maintained by increasing glycogenolysis. ${ }^{7}$ This observation illustrates how ${ }^{31} \mathrm{P}$ nuclear magnetic resonance can be used to study tissue protection. In addition, "well established" ideas can now be directly tested; in this case the demonstration that glycogenolysis proceeds rapidly even though the intracellular $\mathrm{pH}$ decreased to well below 6.3 is unexpected and contrary to established views about the inhibition of phosphofructokinase at low $\mathrm{pH}$.

\section{REACTION FLUXES: IN VIVO ENZYMOLOGY}

A unique aspect of in vivo nuclear magnetic resonance spectroscopy is that the fluxes of intracellular enzyme catalysed reactions can be measured even when the system is in equilibrium or a steady state. Fig. 3 illustrates the method of saturation transfer. ${ }^{8}$ Consider, for example, interconversion of phosphocreatine to adenosine triphosphate and of adenosine triphosphate to inorganic phosphate, catalysed by the enzymes creatine kinase and mitochrondrial adenosine triphosphatase. If in some way we were to "pulse label" the gamma phosphate residue of adenosine triphosphate, this label would be chemically transferred to phosphocreatine and inorganic phosphate, giving an opportunity to measure the appropriate reaction rates. Labelling is achieved by selective irradication of the gamma phosphate of adenosine triphosphate. This perturbs its magnetic (but not chemical) properties and results in eliminating (saturation) the corresponding peak from the spectrum. When this magnetisation is chemically transferred to the phosphorus groups of phosphocreatine and inorganic phosphate the decrease in the intensities of their resonances is used to measure quantitatively the fluxes phosphocreatine $\rightarrow$ adenosine triphosphate and inorganic phosphate $\rightarrow$ adenosine triphosphate. In a beating rat heart the flux through creatine kinase is considerably faster than the rate of adenosine triphosphate synthesis (Table 1), a prediction that could not be tested in vivo before. Comparison of adenosine triphosphate synthesis with oxygen uptake now also makes it possible to study the efficiency of oxidative phosphorylation in vivo.9 This is of particular interest in the examination of cardiomyopathies, hypertrophy, thyrotoxicosis, etc.

\section{Towards the clinic}

It is now possible to carry out the kind of biochemical studies as outlined above on intact tissues and organs of laboratory animals and humans. The key to this 
Table 1 Results of saturation transfer, ${ }^{\star}$ metabolite concentration, $\dagger$ and values for the time constant $T_{1}$ (figures are means $\pm S D$ )

\begin{tabular}{|c|c|c|c|c|}
\hline $\begin{array}{l}P C r \\
\text { ( } \mu \text { mol/g dry weight) }\end{array}$ & $\begin{array}{l}P_{i} \\
(\mu m o l / g \text { dry weight })\end{array}$ & $M^{+} / M^{\circ}(P C r)$ & $M^{+} / M^{\circ}\left(P_{i}\right)$ & $\underset{(s)}{T_{1}\left(P_{i}\right)}$ \\
\hline $28.4 \pm 1 \cdot 7$ & $9.7 \pm 1 \cdot 1$ & $0.32 \pm 0.01$ & $0.64 \pm 0.02$ & $1.9 \pm 0.2$ \\
\hline
\end{tabular}

$\mathrm{PCr}$, phosphocreatine; $\mathrm{P}_{i}$, inorganic phosphate.

^Extent of saturation transfer is expressed as a fraction of the signal intensity with irradiation to high frequency of inorganic phosphate. fConcentrations were determined from the relative areas of the metabolites and calibrated external standard resonances as described in the methods.

approach is to localise the spectroscopic observations to a defined volume element within the subject to be examined. So far, two main methods of localising ${ }^{31} \mathrm{P}$ nuclear magnetic resonance measurements have been used: surface coils ${ }^{10}$ and field profiling (focusing). ${ }^{11}$ These techniques (or a combination of the two) have been used in examining the biochemical energetics of skeletal muscle, brain, heart, liver, and kidney in laboratory animals and of skeletal muscle in humans.

In animal models, for example, the time course of metabolic changes in the heart following respiratory arrest has been examined (Fig. 4). ${ }^{12}$ In haemorrhagic shock, immediate changes in the concentrations of adenosine triphosphate and intracellular $\mathrm{pH}$ are seen in the kidney, ${ }^{13}$ while high energy phosphates are maintained in the brain despite a drastic reduction in blood pressure. ${ }^{14}$ Models for stroke in gerbils ${ }^{15}$ and in a monkey ${ }^{14}$ show regionalised metabolic changes in the brain.

\section{In the clinic}

Since early 1981, it has been possible to examine human muscle metabolism by ${ }^{31} \mathrm{P}$ nuclear magnetic resonance in limbs. ${ }^{16}$ In the clinical magnetic resonance laboratory at the Radcliffe Infirmary, Oxford, we examined patients with disorders of the neuromuscular system and studied the bioenergetics of intact human muscle in normal healthy volun-

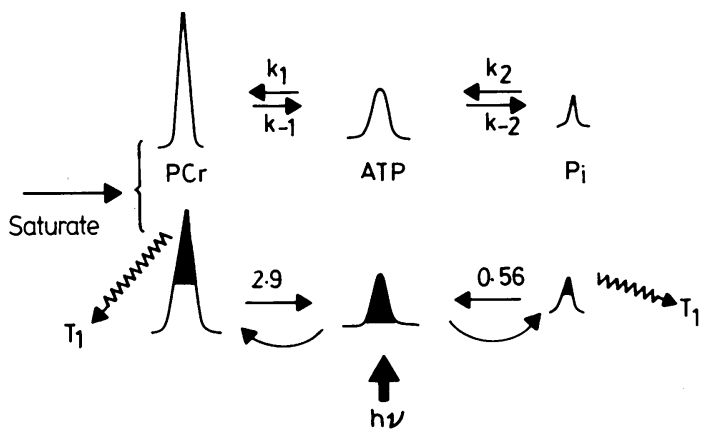

Fig. 3 Schematics of saturation transfer nuclear magnetic resonance for heart muscle. (Results given in Table 1 and reference 8). $P C r$, phosphocreatine; $P_{i}$, inorganic phosphate; $A T P$, adenosine triphosphate. teers. ${ }^{16}$ The concentrations of phosphocreatine, adenosine triphosphate, and inorganic phosphate as well as intracellular $\mathrm{pH}$ in the flexor digitorum superficialis can be measured during rest, dynamic exercise (aerobic and anaerobic), and recovery from exercise (Fig. 5). While there are large variations in the relative amounts of oxidative and glycolytic capacities of different individuals we were able to establish a set of criteria that are characteristic of healthy muscle.

(1) At rest, intracellular $\mathrm{pH}$, and the relative concentrations of phosphocreatine, inorganic phosphate, and adenosine triphosphate are constant.

(2) During aerobic exercise there is a characteristic relation between the decrease in phosphocreatine and intracellular $\mathrm{pH}$, indicating that lactate production becomes significant only after more than $60 \%$ of the phosphocreatine is used up. Surprisingly, intracellular $\mathrm{pH}$ may reach as low a value as 5.9 to $6 \cdot 1$, showing that phosphofructokinase is still partially active at $\mathrm{pH}$ 6.0.

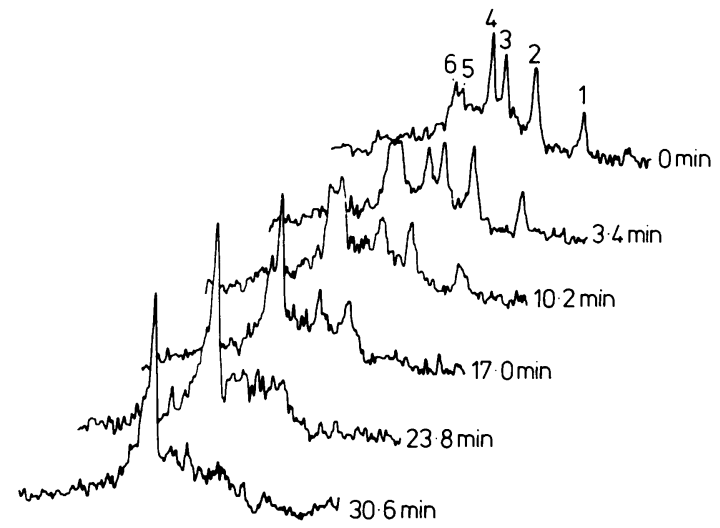

Fig. 4 Series of ${ }^{31} P$ nuclear magnetic resonance spectra of in vivo rat heart recorded as a function of time during respiratory arrest. Each spectrum represents the Fourier transform average of 400 sampling pulses and required 6.8 min for total collection. The times given are the midpoints of each accumulation. Peak assignments: 1 , beta-ATP; 2 , alpha-ATP + alpha-ADP + phosphodiesters; 3 , gamma-ATP + beta-ADP; 4, phosphocreatine; $5, P_{i}+$ 2-phosphate of 2,3-diphosphoglyceratt; 6, 3-phosphate of 2,3-diphosphoglycerate. 


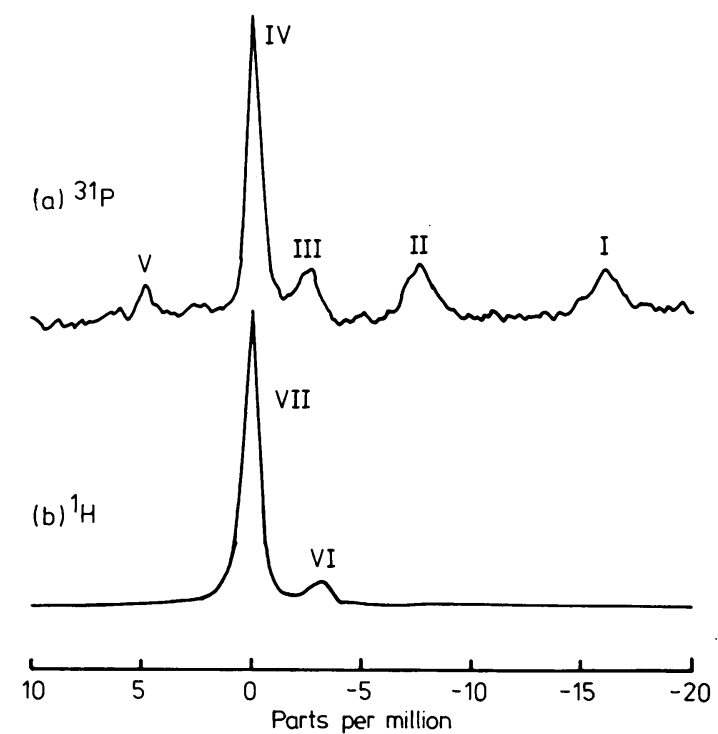

Fig. $5{ }^{31} P$ spectrum $(a)$ and ${ }^{1} H$ spectrum $(b)$ of male subject. Spectrum (a) was collected over $256 \mathrm{~s}$ and $(b)$ over 8 s (1 sampling pulse every $2 \mathrm{~s}$ ). Peak assignments are: I, beta-phosphate of adenosine triphosphate; II, alpha-phosphate of adenosine triphosphate plus NAD+ and NADH; III, gamma-phosphate of adenosine triphosphate; $I V$, phosphocreatine; $V$, inorganic phosphate; VI, triglycerides; VII, water.

(3) There is no metabolic recovery if the muscle is made ischaemic after exercise, implying that glycolysis is switched off as soon as exercise is stopped. Lactic acidosis is not the cause of this and pre- sumably $\mathrm{Ca}^{++}$is needed to maintain the activation of phosphorylase kinase.

(4) During recovery the rate of phosphocreatine resynthesis reflects oxidative capacity while the faster disappearance of inorganic phosphate gives indication about intracellular phosphate redistribution.

(5) The rate of $\mathrm{pH}$ recovery is a measure of $\mathrm{H}^{+}$ export from the muscle cell.

We have already seen abnormal behaviour in all these indices in various pathological states among the 210 patients that have been investigated in Oxford. Table 2 summarises the number of patients we have seen with different conditions.

Primary metabolic lesions-for example, enzyme defects - in the glycolytic pathway ${ }^{3}$ or mitochrondrial dysfunction ${ }^{1718}$ are readily observed. The consequences of dystrophies, of insufficient substrate delivery, can be recorded, ${ }^{19}$ and the effect of various systemic dysfunctions on muscle metabolism can be investigated. In the latter group changes observed in renal failure, diabetes, hypothyroid and hyperthyroid conditions may provide a useful index to evaluate the efficacy of corrective procedures.

Among the many patients with undiagnosed muscle weakness and pain, a group emerged with abnormal control in the coordination of aerobic and anaerobic energy supply. Interestingly, such metabolic disturbances appear to be associated with a postviral condition that can persist for a long period after a severe virus attack (for example, influenza).

In one patient we examined the metabolic consequences of treatment with verapamil and found restoration of some control function possibly associated with intracellular $\mathrm{Ca}^{++}$.

Table 2 Biochemical disorders studied by ${ }^{31 P}$ nuclear magnetic resonance in 203 patients (367 examinations) according to Oxford patient census (May 1981 to March 1983)

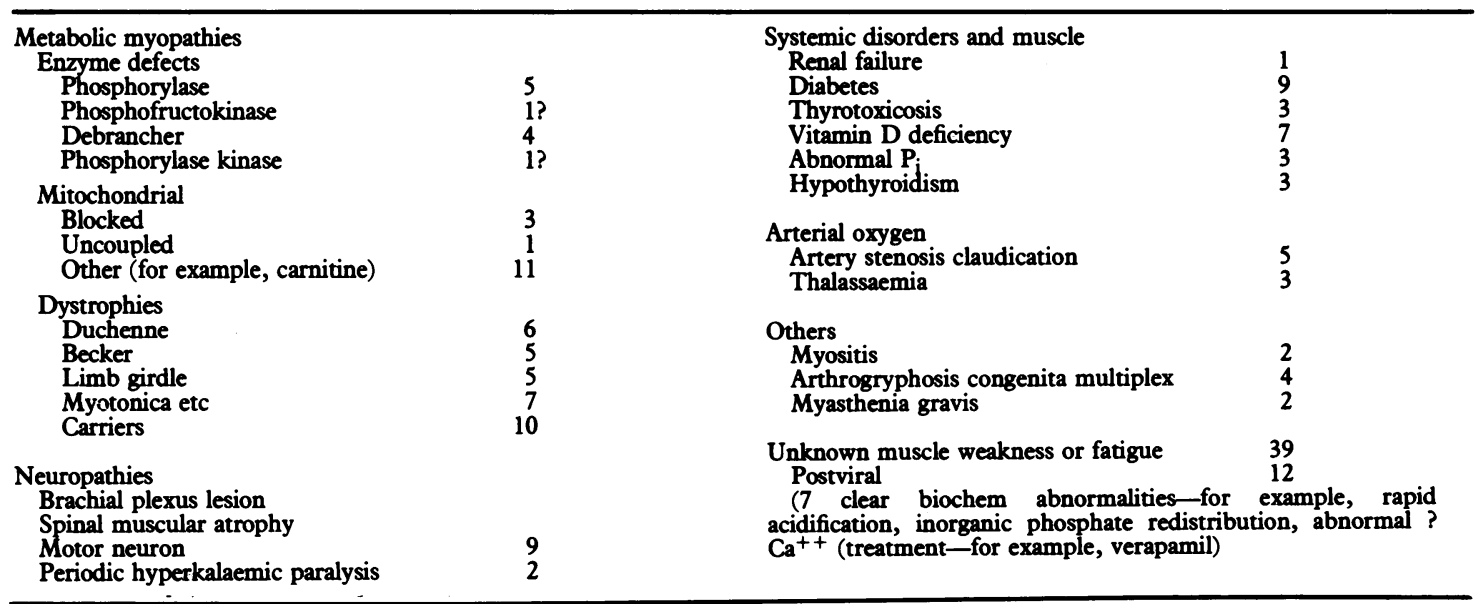




\section{Study of human heart disease}

The technology to observe ${ }^{31} \mathrm{P}$ nuclear magnetic resonance spectra in the human heart is now available and clinical studies will soon begin. It is important that we explore this new technique in relation to existing clinical methods, yet at the same time realise that it gives us a new approach to human investigations. It tells us about the biochemistry of the tissue, albeit at relatively low spatial resolution. Because of these, and because the measurements can be repeated, new tests can be designed to elucidate the basis of the disorder and to develop or monitor treatment. Thus the technique should not be looked on as yet another expensive diagnostic imaging technique but as a way of providing an objective and rational approach to clinical research and patient management. In my view, this technique cannot fail to enhance our understanding of cardiomyopathies or to contribute to clinical evaluations of the salvaging or correction of ischaemic heart disease.

This work was supported by the Medical Research Council, the British Heart Foundation, and a grant from the National Institute of Health (USA).

\section{References}

1 Gadian DG, Radda GK. NMR studies of tissue metabolism. Annu Rev Biochem 1981; 50: 69-83.

2 Gordon RE, Hanley PE, Shaw D, et al. Localization of metabolites in animals using ${ }^{31} \mathrm{P}$ topical magnetic resonance. Nature 1980; 287: 736-8.

3 Ross BD, Radda GK, Gadian DG, Rocker G, Esiri M, Falconer-Smith J. Examination of a case of suspected McArdle's syndrome by ${ }^{31} \mathrm{P}$ nuclear magnetic resonance. N Engl f Med 1981; 304: 1338-42.

4 Steiner RE, Bydder GM, Selwyn A, et al. Nuclear magnetic resonance imaging of the heart. Current status and future prospects. Br Heart $\mathcal{F}$ 1983; 50: 202-8.

5 Bailey IA, Williams SR, Radda GK, Gadian DG. Activity of phosphorylase in total global ischaemia in the rat heart: a phosphorus-31 nuclear magnetic resonance study. Biochem f 1981; 196: 171-8.

6 Garlick PB, Radda GK, Seeley PJ. Studies of acidosis in the ischaemic heart by phosphorus nuclear magnetic resonance. Biochem $\mathcal{F}$ 1979; 184: 547-54.
7 Bailey IA, Radda GK, Seymour A-ML, Williams SR. The effects of insulin on myocardial metabolism and acidosis in normoxia and ischaemia: a ${ }^{31}$ P-NMR study. Biochim Biophys Acta 1982; 720: 17-27.

8 Matthews PM, Bland JL, Gadian DG, Radda GK. The steady state rate of ATP synthesis in the perfused rat heart measured by ${ }^{31} \mathrm{P}$ NMR saturation transfer. Biochem Biophys Res Comomun 1981; 103: 1052-9.

9 Matthews PM, Bland JL, Gadian DG, Radda GK. A ${ }^{31}$ P NMR saturation transfer study of the regulation of creatine kinase in the rat heart. Biochim Biophys Acta 1982; 721: 312-20.

10 Ackerman JJH, Grove TH, Wong GG, Gadian DG, Radda GK. Mapping of metabolites in whole animals by 31P NMR using surface coils. Nature 1980; 283: 167-70.

11 Balaban RS, Gadian DG, Radda GK. A phosphorus nuclear magnetic resonance study of the rat kidney in vivo. Kidney Int 1981; 20: 575-9.

12 Grove TH, Ackerman JJH, Radda GK, Bore PJ. Analysis of rat heart in vivo by phosphorus nuclear magnetic resonance. Proc Natl Acad Sci USA 1980; 77: 299-302.

13 Chan L, Waterton JC, Radda GK. Study of rat kidney in vivo during hypovolaemic shock by ${ }^{31}$ P NMR. Biochem Soc Trans 1981; 239-40.

14 Radda GK, Chan L, Bore PJ, et al. Clinical applications of ${ }^{31}$ P NMR. In: Witcofski RL, Karstaedt N, Partain CL, eds. NMR imaging. North Carolina: The Bowman Gray School of Medicine, 1982: 159-69.

15 Thulborn KR, du Boulay GD, Duchen LM, Radda GK. A ${ }^{31} \mathrm{P}$ nuclear magnetic resonance (NMR) in vivo study of cerebral ischaemia in the gerbil. Fourmal of Cerebral Blood Flow and Metabolism 1982; 2: 299-306.

16 Taylor DJ, Bore PJ, Styles P, Gadian DG, Radda GK. Bioenergetics of intact human muscle. A ${ }^{31} \mathrm{P}$ nuclear magnetic resonance study. Mol Biol Med 1983; 50: in press.

17 Gadian DG, Radda GK, Ross BD, et al. Examination of a myopathy by phosphorus nuclear magnetic resonance. Lancet 1981; ii: 774-5.

18 Radda GK, Bore PJ, Gadian DG, et al. ${ }^{31}$ P NMR examination of two patients with $\mathrm{NADH}-\mathrm{CoQ}$ reductase deficiency. Nature 1982; 295: 608-9.

19 Newman RJ, Bore PJ, Chan L, et al. Nuclear magnetic resonance studies of forearm muscle in patients in Duchenne dystrophy. $\mathrm{Br} \mathrm{Med} \mathcal{F} 1$ 1982; 284: 1072-4.

Requests for reprints to $\mathrm{Dr}$ George $\mathrm{K}$ Radda, Department of Biochemistry, University of Oxford, South Parks Road, Oxford OX1 3QU. 